\title{
Disk-Shaped Amperometric Enzymatic Biosensor for in Vivo Detection of D-serine
}

\author{
David Polcari, ${ }^{\dagger, \perp}$ Annie Kwan, ${ }^{\ddagger}, \perp$ Marion R. Van Horn, ${ }^{\ddagger}$ Laurence Danis, ${ }^{\dagger}$ Loredano Pollegioni, ${ }^{\S}, \|$ \\ Edward S. Ruthazer, ${ }^{*}+\frac{\hbar}{+}$ and Janine Mauzeroll ${ }^{* \dagger}$ \\ ${ }^{\dagger}$ Department of Chemistry, McGill University, 801 Sherbrooke Street West, Montreal, Quebec, Canada H3A 0B8 \\ ${ }^{\ddagger}$ Montreal Neurological Institute, McGill University, 3801 University Street, Montreal, Quebec, Canada H3A 2B4 \\ ${ }^{\S}$ Dipartimento di Biotecnologie e Scienze della Vita, Università degli studi deII’Insubria, via J. H. Dunant 3, 21100 Varese, ltaly \\ "The Protein Factory, Centro Interuniversitario di Biotecnologie Proteiche, Politecnico di Milano, ICRM CNR Milano, and \\ Università degli Studi deII’Insubria, Varese, Italy
}

\section{Supporting Information}

ABSTRACT: At the synapse, D-serine is an endogenous coagonist for the $\mathrm{N}$-methyl-D-aspartate receptor (NMDAR). It plays an important role in synaptic transmission and plasticity and has also been linked to several pathological diseases such as schizophrenia and Huntington's. The quantification of local changes in D-serine concentration is essential to further understanding these processes. We report herein the development of a disk-shaped amperometric enzymatic biosensor for detection of $\mathrm{D}$-serine based on a $25 \mu \mathrm{m}$ diameter platinum disk microelectrode with an electrodeposited poly- $m$-phenylenediamine (PPD) layer and an $R$. gracilis D-amino acid oxidase (RgDAAO) layer. The disk-shaped D-serine biosensor is $1-5$ orders of magnitude smaller than previously reported probes and exhibits a sensitivity of $276 \mu \mathrm{A} \mathrm{cm}^{-2} \mathrm{mM}^{-1}$ with an in vitro detection limit of $0.6 \mu \mathrm{M}$. We demonstrate its usefulness for in vivo applications by measuring the release of endogenous D-serine in the brain of Xenopus laevis tadpoles.

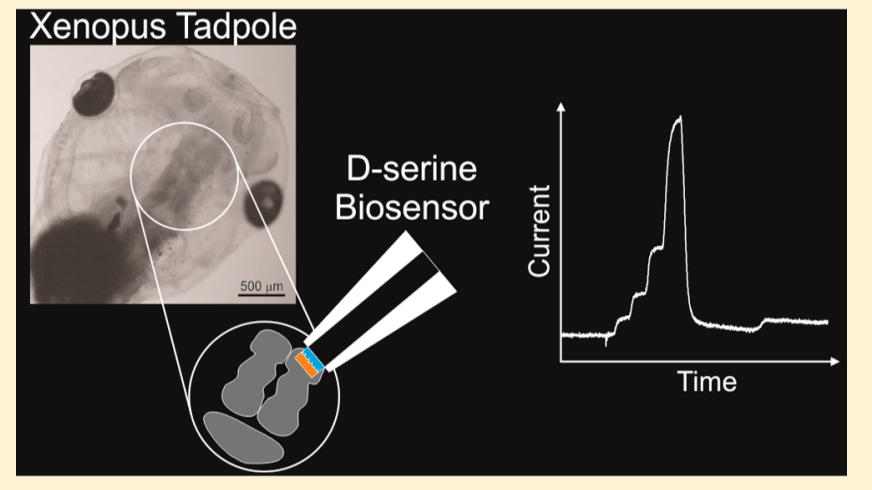

A strocyte-derived D-serine is an endogenous co-agonist for $N$-methyl-D-aspartate receptors (NMDARs) at synapses in many regions of the mature brain, including the cerebral cortex, hippocampus, and cerebellum. ${ }^{1,2}$ NMDARs, a member of the glutamate receptor family, play an especially important role in basal synaptic transmission and as a critical mediator of many forms of synaptic plasticity. ${ }^{3,4}$ They have also been important clinical therapeutic targets in psychiatric disorders such as schizophrenia and depression, ${ }^{5,6}$ as well as neurodegenerative disorders like Huntington's disease ${ }^{7}$ and amyotrophic lateral sclerosis (ALS). ${ }^{8,9}$ Activation of the NMDAR requires concurrent binding of its primary ligand glutamate, which is the most common excitatory neurotransmitter in the vertebrate central nervous system (CNS), ${ }^{10}$ at a selective glutamate binding site, ${ }^{11}$ together with binding of a co-agonist, either glycine or D-serine, at the so-called glycine binding site. Thus, astrocyte release of $\mathrm{D}$-serine can modulate excitatory neuronal transmission and synaptic plasticity. ${ }^{12}$

In contrast to transmitter release by neurons, which has been extensively studied and characterized at the level of the underlying molecular machinery, the mechanisms responsible for astrocyte release of $\mathrm{D}$-serine remain controversial and are less well understood. One reason for this is the fact that "gliotransmission" is thought mainly to exert modulatory influences on neuronal communication and to be less temporally restricted than the rapid vesicular neurotransmission between synaptic pairs of neurons. In contrast to electrically excitable neurons, glial cells do not exhibit a rapid depolarization of their membrane potential in response to neurochemical stimulation that can be measured using microelectrodes. However, a number of studies using calcium-sensitive fluorescent dyes have revealed cell-wide calcium transients in glial astrocytes which may initiate the release of gliotransmitters like D-serine. ${ }^{13,14}$ Moreover, rapid propagation of calcium elevations between astrocytes in culture has been observed, demonstrating the ability to communicate with each other through a network of gap junctional connections. ${ }^{15}$ Recent calcium imaging studies further suggest that astrocytes may exhibit very local calcium transients that can influence synaptic efficacy at nearby neuronal contacts. ${ }^{16,17}$ Thus, improving the ability to precisely localize extracellular changes in D-serine concentration at the cellular or even

Received: December 18, 2013

Accepted: March 6, 2014

Published: March 7, 2014 
subcellular level constitutes a critical step toward better characterizing this fundamental aspect of neuron-glia communication.

Previous efforts to measure D-serine levels in situ have relied primarily on local sampling by microdialysis. ${ }^{18,19}$ Highly specific detection of $\mathrm{D}$-serine in perfusion samples from the brain can be obtained using high-performance liquid chromatography $(\text { HPLC })^{20,21}$ or by fluorescence-based capillary electrophoresis (CE) of fluorescently derivatized distillates of cerebrospinal fluid (CSF). ${ }^{22,23}$ In combination with implantable microdialysis probes, limited spatial and temporal information can be obtained by these approaches. Several reports have shown that significantly improved spatiotemporal resolution can be provided by the use of enzymatic amperometric biosensors. ${ }^{24,25}$ One method of detection of D-serine using such probes is based on the production of $\mathrm{H}_{2} \mathrm{O}_{2}$ during the enzymatic degradation of D-serine by D-amino acid oxidase (DAAO). ${ }^{26}$ These biosensors can be fabricated small enough to insert directly into brain tissue of interest. Because D-amino acids, with the exception of D-serine, are extremely rare in the $\mathrm{CNS},{ }^{27}$ this method offers reasonable selectivity and spatial accuracy limited only by the geometry of the functionalized probe tip itself. Previously described D-serine biosensors have proven useful for in vivo applications ${ }^{28,29}$ but have been based on functionalized tips too large to allow measurements on the scale of individual cells. Here we report the development of a $25 \mu \mathrm{m}$ diameter disk amperometric enzymatic biosensor that is $1-5$ orders of magnitude smaller than previously reported probes and which displays increased sensitivity for D-serine detection. We further demonstrate its usefulness for in vivo applications by measuring the unprecedented evoked release of endogenous D-serine in the brain of stage 48 albino Xenopus laevis tadpoles.

\section{EXPERIMENTAL SECTION}

Chemicals. Ascorbic acid, dopamine, D-serine, ferrocenemethanol $\left(\mathrm{FcCH}_{2} \mathrm{OH}\right)$, hydrogen peroxide, L-glutamate, $m$ phenylenediamine, ethyl 3-aminobenzoate methanesulfonate (MS222), and serotonin were purchased from Sigma-Aldrich (Oakville, Canada). Calcium chloride, calcium nitrate, glucose, HEPES, magnesium chloride, magnesium sulfate, potassium chloride, sodium chloride, and sodium bicarbonate were purchased from VWR (Mississauga, Canada). Tetrodotoxin (TTX), cyclothiazide, and 2-amino-3-(hydroxy-5-methyl-isoxazol-4-yl) propanoic acid (AMPA) were purchased from R\&D Systems, Inc. (Minneapolis, U.S.A.). Other chemical reagents were purchased from Fisher Scientific (Ottawa, Canada), unless otherwise noted.

Enzyme Preparation. Recombinant $R$ gracilis D-amino acid oxidase (RgDAAO, EC 1.4.3.3) was overexpressed in $E$. coli cells and purified to homogeneity as previously reported. ${ }^{30}$ The final enzyme solution was concentrated to $50 \mathrm{mg} / \mathrm{mL}$ protein in PBS (0.01 M, pH 7.4) with $1 \%$ glycerol and $25 \mathrm{mg} /$ $\mathrm{mL}$ bovine serum albumin (BSA); pure RgDAAO had a specific activity of $75 \pm 7 \mathrm{U} / \mathrm{mg}$ protein on D-serine as substrate based on amperometric assay.

Electrochemical Measurements. Electrochemical measurements were performed using either an Electrochemical Probe Scanner 3 (Heka Elektronik, Lambrecht, Germany) or an Axopatch 200B amplifier (Molecular Devices, Sunnyvale, U.S.A.). All potentials were recorded relative to a chloridized silver wire (in house) quasi-reference electrode. In vitro calibrations in $0-50 \mu \mathrm{M}$ D-serine in PBS (0.01 M, pH 7.4) were obtained using chronoamperometry $(10 \mathrm{~min}, 500 \mathrm{mV})$ with a three-electrode setup (Pt wire counter electrode). In vivo calibrations were also performed using chronoamperometry after insertion into the optic tectum of tadpoles. In vivo standard solutions were made in external artificial cerebrospinal fluid (ACSF) containing $115 \mathrm{mM} \mathrm{NaCl}, 2 \mathrm{mM} \mathrm{KCl}, 3 \mathrm{mM}$ $\mathrm{CaCl}_{2}, 3 \mathrm{mM} \mathrm{MgCl} 2,5 \mathrm{mM}$ HEPES, $10 \mathrm{mM}$ glucose, $1 \mu \mathrm{M}$ TTX (to prevent neuronal activity), at $\mathrm{pH}$ 7.4. All in vivo measurements were performed using a two-electrode system.

Preparation of Biosensor Backbone: $25 \mu \mathrm{m}$ Pt Disk Microelectrode. Microelectrodes were fabricated by initially pulling a soda-lime glass capillary (Hilgenberg $\mathrm{GmbH}$, Malsfeld, Germany) using a P-2000 micropipette puller (Sutter Instruments, Novato, CA). A $25 \mu \mathrm{m}$ diameter Pt wire (Goodfellow, Huntington, U.K.) was then inserted into the capillary and sealed using a PC-10 vertical pipette puller (Narishige, Japan) under vacuum. The $\mathrm{Pt}$ wire was electrically connected to a copper wire using silver epoxy (H20E, Epo-Tek, Billerica, U.S.A.), which was then cured at $150{ }^{\circ} \mathrm{C}$ for $30 \mathrm{~min}$. To increase mechanical stability, the microelectrode was inserted into a larger borosilicate glass capillary (Sutter Instruments, Novata, U.S.A.), with an overlap of $1.5 \mathrm{~cm}$. A gold connector (HEKA Elektronik, Lambrecht, Germany) was soldered onto the copper wire, and junctions were sealed with epoxy glue (Henkel Canada, Mississauga, Canada). The microelectrode tip was mechanically polished (400 rpm, 4000 grit Silicon carbide grinding paper, $15 \mathrm{~min}$ ) using a TegraPol-25 grinder/polisher (Struers Ltd., Mississauga, Canada) until the Pt wire was exposed as a disk. The microelectrode was then washed in 18.2 $\mathrm{M} \Omega$ water, $70 \%$ ethanol, and acetone in preparation for subsequent steps. The diameter of the microelectrode was characterized using cyclic voltammetry $(3$ cycles, $-100 \mathrm{mV}$ to $+500 \mathrm{mV}, 5 \mathrm{mV} \mathrm{s}^{-1}$ ) in $1 \mathrm{mM} \mathrm{FcCH}_{2} \mathrm{OH}$ (in $0.1 \mathrm{M} \mathrm{KCl}$ ). The $\mathrm{RG}$ of the microelectrode, defined as the ratio of the radius of the entire microelectrode (glass $+\mathrm{Pt}$ wire) to that of the $\mathrm{Pt}$ metal wire, was determined by optical microscopy using a customized Axio Vert.A1 inverted microscope (Zeiss, Oberkochen, Germany).

Addition of Polymer and Enzyme Layer. A layer of 100 $\mathrm{mM}$ poly-meta-phenylenediamine (PPD) in PBS (0.01 M, pH 7.4) was electropolymerized onto the Pt surface using cyclic voltammetry $\left(15\right.$ cycles, 0 to $\left.+1000 \mathrm{mV}, 100 \mathrm{mV} \mathrm{s}^{-1}\right)$. The PPD-modified microelectrode was then immersed tip-up in a $0.5 \mu \mathrm{L}$ droplet of final enzyme solution, formed at the aperture of a micropipette tip. The enzyme meniscus was allowed to evaporate, leaving approximately $2 \mathrm{U}$ of adsorbed enzyme on the PPD-modified microelectrode. The biosensor was then placed in a sealed glass chamber containing $10 \mathrm{~mL}$ of glutaraldehyde $\left(50 \% \mathrm{v} / \mathrm{v}\right.$ in $\left.\mathrm{H}_{2} \mathrm{O}\right)$, as a source of vapor, to crosslink the RgDAAO for $10 \mathrm{~min}$.

Null biosensors were produced using the exact same procedure described above, with the exception of the active RgDAAO layer. Instead, the PPD-modified microelectrode was immersed in a solution of $400 \mathrm{mg} / \mathrm{mL}$ BSA in PBS $(0.01 \mathrm{M}$, $\mathrm{pH}$ 7.4) and crosslinked using glutaraldehyde.

Tadpole Preparation and In Vivo Measurements. Brains from stage 48 albino Xenopus laevis tadpoles were dissected out following anesthesia by immersion in $0.02 \%$ MS222 0.1× MBS-H (Modified Barth's Saline with HEPES) rearing solution and placed in ACSF. The rearing solution was composed of $880 \mu \mathrm{M} \mathrm{NaCl}, 10 \mu \mathrm{M} \mathrm{KCl}, 24 \mu \mathrm{M} \mathrm{NaHCO}_{3}, 8.2$ $\mu \mathrm{M} \mathrm{MgSO}_{4} \cdot\left(6 \mathrm{H}_{2} \mathrm{O}\right), 3.3 \mu \mathrm{M} \mathrm{Ca}\left(\mathrm{NO}_{3}\right)_{2} \cdot\left(4 \mathrm{H}_{2} \mathrm{O}\right), 4.1 \mu \mathrm{M}$ $\mathrm{CaCl}_{2} \cdot\left(2 \mathrm{H}_{2} \mathrm{O}\right)$, and $100 \mu \mathrm{M}$ HEPES, adjusted to $\mathrm{pH}$ 7.4. To expose the ventricular and pial surfaces of the optic tectum, 

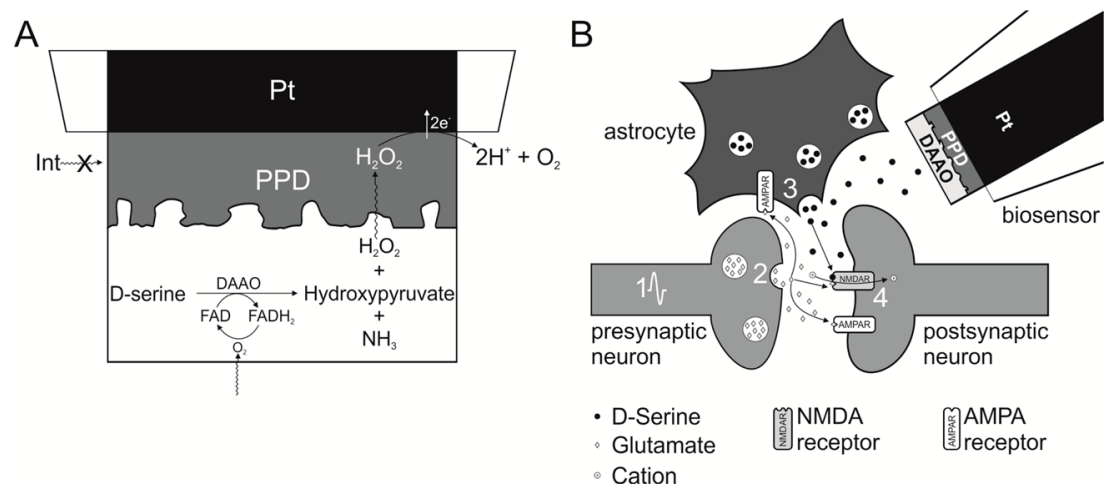

Figure 1. Principle of D-serine detection using a disk-shaped amperometric enzymatic biosensor. (A) D-serine reacts with immobilized RgDAAO, degrading into hydroxypyruvate and ammonia. Molecular oxygen oxidizes $\mathrm{FADH}_{2}$ back to $\mathrm{FAD}$, producing equimolar $\mathrm{H}_{2} \mathrm{O}_{2}$, which then diffuses through the interference blocking PPD layer and is oxidized at the biased Pt surface. (B) Schematic representation of the in vivo measurement environment within the brain (not to scale). The disk-shaped biosensor is used to measure evoked release of D-serine from astrocytes.

brains were fileted down the midline and pinned to a piece of Sylgard, which was submerged in a recording chamber perfused with fresh ACSF.

A Narishige micromanipulator was used to position the biosensor on the ventricular surface of the brain adjacent to the tectal cell body layer. The brain was then allowed to stabilize for $20 \mathrm{~min}$ in this configuration. D-serine release was stimulated by perfusing ACSF containing $100 \mu \mathrm{M}$ AMPA with $50 \mu \mathrm{M}$ cyclothiazide to activate AMPA-type glutamate receptors and to block receptor desensitization, respectively.

All animal experiments were approved by the Montreal Neurological Institute Animal Care Committee.

Data Analysis. Data are presented as mean \pm standard error of the mean (S.E.M). Oxidation current values are presented as blank corrected currents. The number of data points is defined as $n$. The limit of detection (LOD) is defined as 3 times the standard deviation of the blank divided by the slope of the regression line. The limit of quantitation (LOQ) is defined as 10 times the standard deviation of the blank divided by the slope of the regression line. Data sets were analyzed using either Excel 2010 (Microsoft Office) or Matlab R2013a (Mathworks, Natick, U.S.A.).

\section{RESULTS AND DISCUSSION}

Principle of Detection of D-Serine Release in the Brain. An enlarged schematic of the biosensor tip is shown in Figure 1A. It consists of three components including an RgDAAO enzyme layer (light gray) to react with D-serine, a PPD polymer layer (dark gray) to block out large electroactive interferences, and a platinum surface (black) used for the oxidation of $\mathrm{H}_{2} \mathrm{O}_{2}$. Detection of D-serine using the biosensor relies on the stoichiometric production of $\mathrm{H}_{2} \mathrm{O}_{2}$ during the enzymatic degradation of $\mathrm{D}$-serine. ${ }^{26}$ Upon binding of $\mathrm{D}$-serine with $\mathrm{RgDAAO}$, a reductive half-reaction occurs, leading to the production of hydroxypyruvate and ammonia, along with reduced flavin adenine dinucleotide $\left(\mathrm{FADH}_{2}\right)$. Subsequently, an oxidative half-reaction with molecular oxygen occurs to produce $\mathrm{FAD}$ and produces an equimolar amount of $\mathrm{H}_{2} \mathrm{O}_{2}$ (Scheme 1). $\mathrm{H}_{2} \mathrm{O}_{2}$ then diffuses through the PPD layer and is oxidized at the biased Pt surface (Scheme 2).

Using this detection principle in combination with D-serine calibration methods, the local concentration of D-serine can be determined in vivo.

Figure $1 \mathrm{~B}$ shows a schematic of the in vivo measurement environment (not to scale), where the disk-shaped biosensor is
Scheme 1

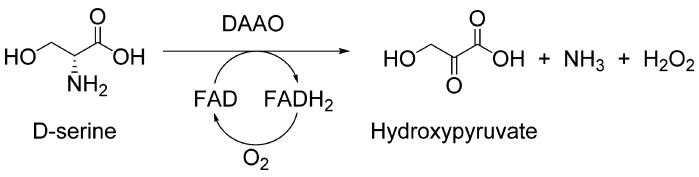

Scheme 2

$$
\mathrm{H}_{2} \mathrm{O}_{2} \stackrel{\mathrm{Pt}(500 \mathrm{mV})}{\longrightarrow} 2 \mathrm{H}^{+}+\mathrm{O}_{2}+2 \mathrm{e}^{-}
$$

inserted into the optic tectum of the tadpole. The proposed release of D-serine consists of four steps, as has been previously described. $^{31-34}$ (1) An action potential reaches the axon terminal of the presynaptic neuron, (2) triggering the release of the neurotransmitter, glutamate. (3) Glutamate, which is an agonist for both AMPA and NMDA receptors, causes AMPA receptors to activate. Activation of the AMPA receptors present on the astrocyte triggers a cascade leading to the release of $\mathrm{D}$ serine. (4) Both glutamate and D-serine, which are co-agonists of the NMDAR at synapses, bind and activate it, allowing cation influx which depolarizes the postsynaptic neuron. Detection of local release of $\mathrm{D}$-serine during this process requires a biosensor that is both highly sensitive and geometrically appropriate to allow sampling with precise spatial resolution. For this reason, the disk-shaped biosensor was required.

Biosensor Geometry. Previously reported D-serine biosensors capable of performing in vivo measurements ${ }^{29,35-37}$ mostly display a cylindrical geometry (e.g., $25 \mu \mathrm{m}$ diameter and $150 \mu \mathrm{m}$ long) several times larger than the brain cells targeted in tadpoles. The cell body of a large cortical pyramidal neuron is approximately $25 \mu \mathrm{m}$ in diameter. The entire brain structure studied herein, the optic tectum in a stage 48 Xenopus laevis tadpole, measures less than $200 \mu \mathrm{m}$ along its mediolateral axis. Although these cylindrical biosensors have a large surface area and relatively good sensitivity, their size and geometry make it difficult to decipher cell body response versus peripheral processes, specific layers of the tectum, or to determine if the observed response originated within the optic tectum. The developed disk-shaped biosensor provides a useful solution to this problem because it can be accurately positioned within the optic tectum, effectively acting as a spatially targeted probe.

To avoid bending previously reported in cylindrical biosensors and improve manipulation in vivo, the configuration 
of the disk-shaped biosensor seals the entire metal wire in a glass matrix, as shown in Figure 2A. The side view optical

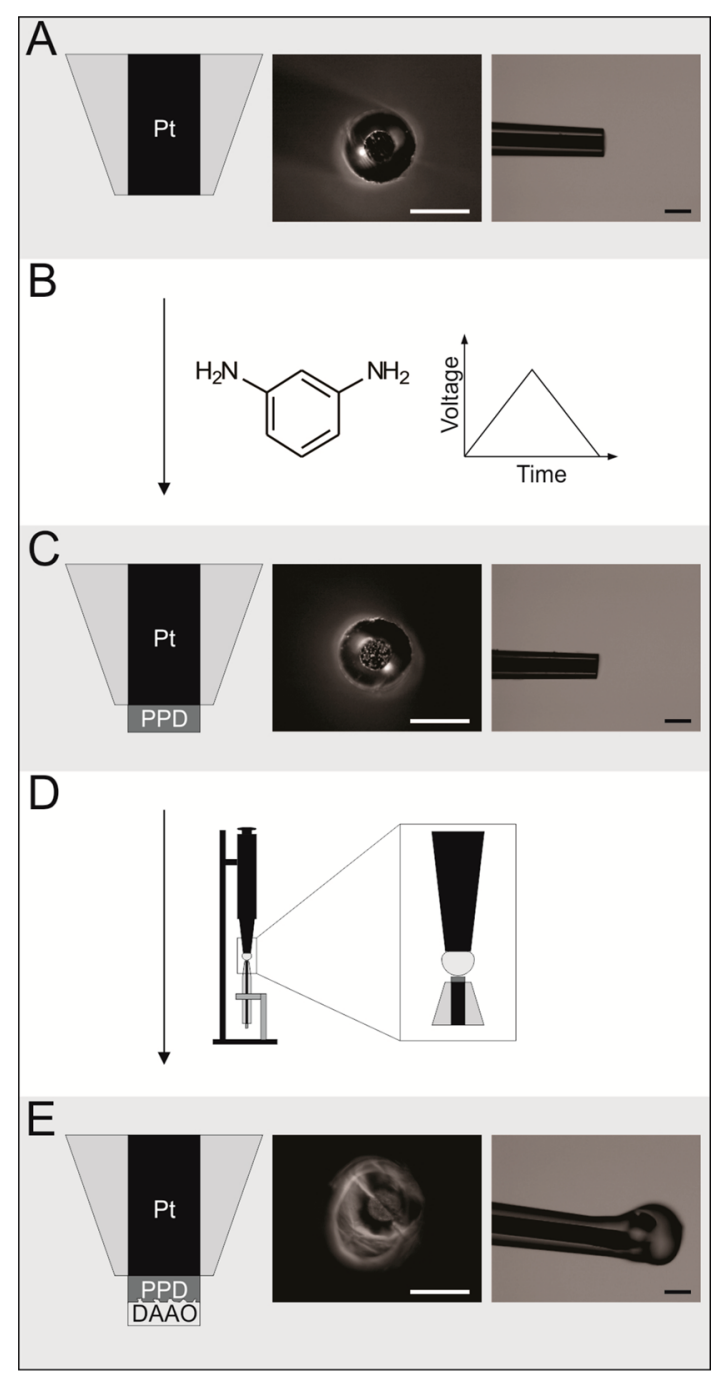

Figure 2. Fabrication of a $25 \mu \mathrm{m}$ diameter disk-shaped amperometric enzymatic biosensor. (A) $25 \mu \mathrm{m}$ Pt disk microelectrode tip. Top and side view optical micrographs show a Pt core centered within a thin insulating glass sheath. (B) A PPD layer is electropolymerized onto the Pt surface using cyclic voltammetry. (C) Disk microelectrode tip with an electrodeposited PPD layer. Optical micrographs confirm successful addition of PPD. (D) RgDAAO is deposited using a micropipette. The enzyme layer is crosslinked using glutaraldehyde vapors, producing a bulb at the tip of the biosensor. (E) Complete biosensor assembly. Scale bars represent $50 \mu \mathrm{m}$.

micrograph shows a $25 \mu \mathrm{m}$ diameter Pt wire surrounded by a thin glass sheath. The RG of the Pt microelectrode was $2.6 \pm$ $0.1(n=15)$ as determined from optical microscopy (see SI; Figure S1). Biosensors fabricated from microelectrodes having such RGs were mechanically stable while retaining the desired spatial resolution for in vivo measurements in tadpoles. Successful exposure of the Pt wire during mechanical polishing is demonstrated by the top view optical micrograph in Figure $2 \mathrm{~A}$, which shows a centered Pt wire surrounded by a thin glass sheath. Following electropolymerization of PPD onto the Pt surface using cyclic voltammetry (Figure $2 \mathrm{~B}$ ), the polymer layer is not thick enough to be observed from the side view optical micrograph (Figure 2C). Successful electrodeposition of PPD is noticeable from the top view optical micrograph (Figure 2C) based on the granular appearance of the microelectrode and is further confirmed by cyclic voltammetry using $\mathrm{FcCH}_{2} \mathrm{OH}$, where the initial faradaic behavior of the bare $\mathrm{Pt}$ surface changed to an ohmic behavior following PPD electropolymerization (see SI; Figure S2). The RgDAAO enzyme layer is adsorbed over PPD by immersion in a stable meniscus of final enzyme solution formed at a micropipette (Figure 2D). Following immobilization of $\mathrm{RgDAAO}$ using glutaraldehyde vapors, a stable enzyme-PPD matrix is formed. ${ }^{38,39}$ The immobilized enzyme was yellow in color, meaning it was in its holoenzyme form. The full biosensor assembly is shown in Figure 2E. The diameter of the final disk-shaped biosensor (approximately $80 \mu \mathrm{m}$ ) is suitable for in vivo studies in the optic tectum of tadpoles. Practically, these sensors can be recycled easily by polishing for $5 \mathrm{~min}$ to regenerate the original $25 \mu \mathrm{m}$ Pt backbone. A new biosensor can be fabricated by replenishing the surface chemistry within $1 \mathrm{~h}$, greatly diminishing total fabrication time and decreasing the total fabrication costs.

Biosensor Selectivity. Insertion of a biased biosensor into living tissue severely complicates the sample matrix due to the presence of other oxidizable molecules, or interferences. Several studies have reported the use of polymer membranes to prevent biological interferences from reaching an electrode surface. ${ }^{40}$ More specifically, PPD has been shown to be particularly effective in excluding ascorbic acid, a highly desirable characteristic for this biosensor. ${ }^{41,42}$ PPD works using the size exclusion principle, whereby relatively large molecules such as ascorbic acid and dopamine are unable to diffuse through the polymer membrane, which remains permeable to $\mathrm{H}_{2} \mathrm{O}_{2}$, a small molecule.

In order to verify that oxidation currents recorded in vivo were in fact related to oxidation of $\mathrm{H}_{2} \mathrm{O}_{2}$, chronoamperometric measurements were performed without and with a PPD layer, in solutions containing physiological concentrations of several possible interfering molecules that could be oxidized at 500 $\mathrm{mV}$. These molecules included ascorbic acid (AA), dopamine (DA), 3,4-dihydroxyphenylacetic acid (DOPAC), serotonin (5HT), 5-hydroxyindoleacetic acid (5-HIAA), homovanillic acid (HVA), and glutamate (GLUT). ${ }^{43}$ The recorded oxidation currents of these interferences at a bare $25 \mu \mathrm{m} \mathrm{Pt}$ disk microelectrode (without PPD) are shown by black bars in Figure 3A. HVA shows a relatively low oxidation current, whereas glutamate produced no detectable current. Consequently, even at relatively high concentrations in the sample matrix of the brain, the contributions of these two molecules to measurements would be negligible. The same cannot be stated for the other five interferences tested, especially for AA, which produced a current greater than $230 \mathrm{pA}$. Following electrodeposition of PPD onto the Pt disk microelectrode, the recorded oxidation decreased significantly for all investigated electroactive interferences (Figure 3A, white bars). For AA, DA, 5-HT, 5-HIAA, and DOPAC, the observed percent decrease in oxidation current corresponded to $77.6 \pm 0.7,71 \pm 2,99.1 \pm$ 0.5 , $99.9 \pm 0.1$, and $92 \pm 1$, respectively $(n=5)$. These decreases confirm that the PPD layer can effectively block out electroactive interferences.

However, the physical presence of a PPD layer also hinders the diffusion of $\mathrm{H}_{2} \mathrm{O}_{2}$ toward the Pt surface. Consequently, it is important to verify that $\mathrm{H}_{2} \mathrm{O}_{2}$ can be detected at a sufficiently low detection limit. Calibration curves obtained in standard solutions of $\mathrm{H}_{2} \mathrm{O}_{2}(2.5-25 \mu \mathrm{M})$ without and with PPD showed 


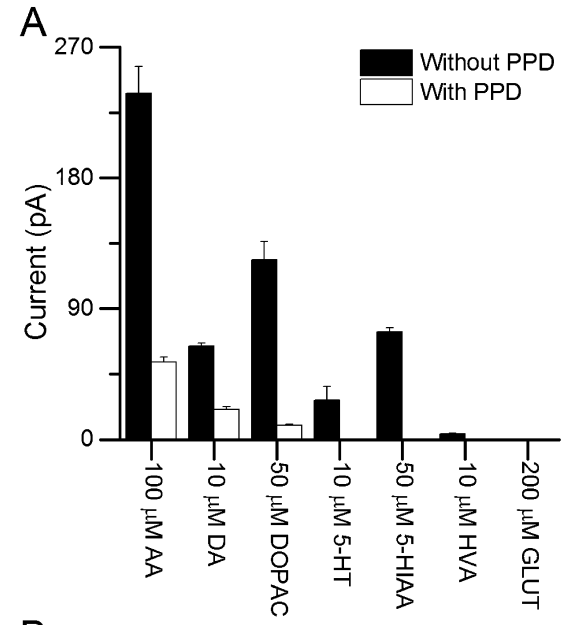

B

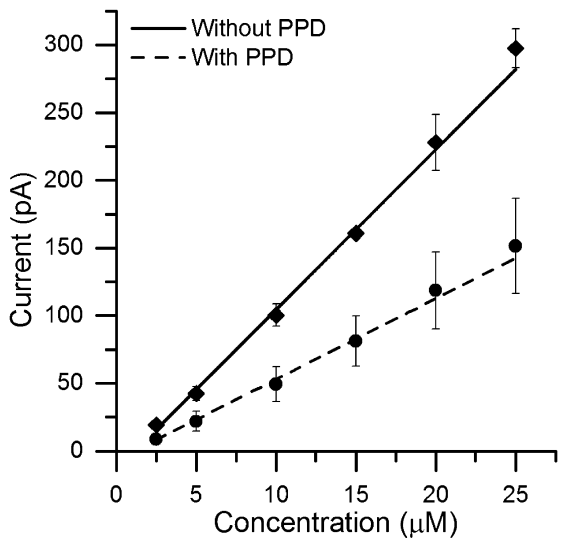

Figure 3. Permeability of the biosensor before and after electrodeposition of a permselective PPD layer. (A) Biosensor response toward ascorbic acid (AA), dopamine (DA), 3,4-dihydroxyphenylacetic acid (DOPAC), serotonin (5-HT), 5-hydroxyindoleacetic acid (5-HIAA), homovanillic acid (HVA), and glutamate (GLUT), without (black) and with (white) PPD layer. (B) Calibration curves for $0-25 \mu \mathrm{M} \mathrm{H}_{2} \mathrm{O}_{2}$ without (solid) and with (dashed) PPD layer ( $n=$ $3)$. Linear regression equation without PPD: $I(\mathrm{pA})=12 \times C(\mu \mathrm{M})-$ 19; $R^{2}=0.992 ; \mathrm{LOD}=0.2 \pm 0.1 \mu \mathrm{M} ; \mathrm{LOQ}=0.8 \pm 0.2 \mu \mathrm{M}$. Linear regression equation with PPD: $I(\mathrm{pA})=6 \times C(\mu \mathrm{M})-11 ; R^{2}=0.995$; $\mathrm{LOD}=0.21 \pm 0.03 \mu \mathrm{M}$; LOQ $=0.7 \pm 0.1 \mu \mathrm{M}$. Currents were measured at constant potential $(500 \mathrm{mV})$.

a $51 \pm 9 \%(n=3)$ decrease in oxidation current (Figure 3B). The LOD of $\mathrm{H}_{2} \mathrm{O}_{2}$ with a PPD-modified microelectrode was $0.2 \mu \mathrm{M}$, which confirms that although the permselective PPD layer slightly hinders its diffusion toward the Pt surface, it is still possible to detect $\mathrm{H}_{2} \mathrm{O}_{2}$.

In Vitro Characterization of Biosensor. The experimental disk-shaped biosensor was characterized in vitro using standard solutions of D-serine in PBS. Figure 4 shows a calibration curve for 2.5-50 $\mu \mathrm{M}$ D-serine, which is a concentration range relevant for in vivo tadpole studies. For comparison purposes, a calibration curve was obtained for both the experimental disk-shaped biosensor and a commercially available cylindrical biosensor (DSER probe; Sarissa Biomedical). In order to correct for the different geometries of both biosensors, recorded oxidation currents were normalized for surface area. Consequently, the values displayed in Figure 4 are shown in terms of current density $\left(\mu \mathrm{A} \mathrm{cm}^{-2}\right)$. The slopes of the regression lines shown demonstrate that although the commercial cylindrical biosensor has a 41 -fold greater surface

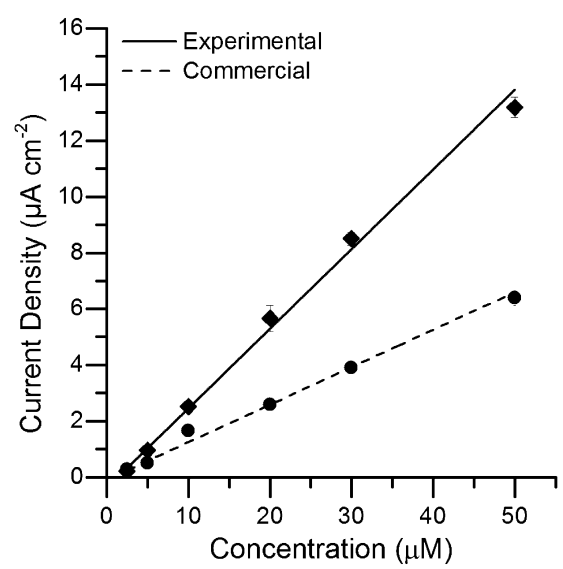

Figure 4. In vitro characterization and comparison of D-serine biosensor. Response toward 0-50 $\mu \mathrm{M}$ D-serine was measured using a commercially available cylindrical biosensor (dashed line; $n=5$ ) and the experimental disk-shaped biosensor (solid line; $n=3$ ). Linear regression equation for the experimental disk-shaped biosensor: $J(\mu \mathrm{A}$ $\left.\mathrm{cm}^{-2} \mu \mathrm{M}^{-1}\right)=0.3 \times C(\mu \mathrm{M})-0.2 ; R^{2}=0.995 ; \mathrm{LOD}=0.6 \pm 0.1 \mu \mathrm{M}$; $\mathrm{LOQ}=2.1 \pm 0.2 \mu \mathrm{M}$. Linear regression equation for the commercial cylindrical biosensor: $J\left(\mu \mathrm{A} \mathrm{cm}^{-2} \mu \mathrm{M}^{-1}\right)=0.1 \times C(\mu \mathrm{M})+0.1 ; R^{2}=$ $0.995 ; \mathrm{LOD}=0.007 \pm 0.002 \mu \mathrm{M}$; LOQ $=0.02 \pm 0.01 \mu \mathrm{M}$. Currents were measured at constant potential $(500 \mathrm{mV})$.

area, the experimental disk-shaped biosensor has a higher sensitivity to D-serine. A comprehensive comparison with all previously reported D-serine biosensors is shown in Table 1. Although the experimental disk-shaped biosensor has a geometry 1-5 orders of magnitude smaller than other probes, it shows at minimum a $30 \%$ higher sensitivity. The improved biosensor sensitivity is likely related to its disk-shaped geometry, which maximizes the ratio of enzymatic to amperometric reaction surface areas as well as the enhanced specific activity of pure RgDAAO $(75 \pm 7 \mathrm{U} / \mathrm{mg}$ protein on Dserine as substrate), as compared to other enzyme homologues (e.g., pig kidney DAAO; $1.5 \mathrm{U} / \mathrm{mg}$ protein).

In Vivo Measurements in Tadpoles. Following characterization and calibration of the experimental disk-shaped biosensor, chronoamperometric measurements were performed in the optic tectum of tadpoles $(n=5)$. A representative response is shown in Figure 5. After positioning in the optic tectum, a $500 \mathrm{mV}$ potential was applied, and the biosensor was allowed a $20 \mathrm{~min}$ stabilization period in ACSF. Standard Dserine solutions in ACSF $(0-30 \mu \mathrm{M})$ were then flowed into the sample chamber. Measurement of D-serine concentration standards washed on in this configuration produced a linear response (Figure 5 inset) with a limit of detection of $0.6 \pm 0.1$ $\mu \mathrm{M}$ and a limit of quantitation of $2.1 \pm 0.2 \mu \mathrm{M}(n=3)$. The sensitivity of the disk-shaped biosensor in vivo was $279 \pm 21$ $\mu \mathrm{A} \mathrm{cm}^{-2} \mathrm{mM}^{-1}(n=3)$, demonstrating the functionality of the biosensor within both in vitro and in vivo environments. Following in vivo calibration, the biosensor remained inside the optic tectum until the current response returned back to the original steady-state level in ACSF ( $\sim 10 \mathrm{~min})$. The AMPA-type glutamate receptor agonist AMPA, along with cyclothiazide to block receptor desensitization, was then added to the perfusion, producing an increase in current measured at the biosensor, which reached a plateau within $2 \mathrm{~min}$. This increase in current represents the release of endogenous D-serine in response to receptor activation and constitutes the first time to our knowledge that release of endogenous $\mathrm{D}$-serine has been 
Table 1. Comparison of Biosensor Properties with Previously Reported D-serine Biosensors

\begin{tabular}{|c|c|c|c|c|c|}
\hline source & geometry $^{a}(\mu \mathrm{m})$ & surface area $\left(\mu \mathrm{m}^{2}\right)$ & surface area ratio ${ }^{b}$ & sensitivity $\left(\mu \mathrm{A} \mathrm{cm}{ }^{-2} \mathrm{mM}^{-1}\right)$ & LOD $(\mu \mathrm{M})$ \\
\hline experimental & disk (25) & 491 & 1 & $276 \pm 6(n=3)$ & 0.6 \\
\hline Vasylieva et al. ${ }^{37}$ & cylindrical $(25 \times 150)$ & 12272 & 25 & $212 \pm 119(n=19)$ & 0.0008 \\
\hline commercial & cylindrical $(25 \times 250)$ & 20126 & 41 & $128 \pm 5(n=5)$ & 0.007 \\
\hline Pernot et al. $^{29}$ & cylindrical $(25 \times 150)$ & 12272 & 25 & $87 \pm 27(n=18)$ & 0.016 \\
\hline Zain et al. ${ }^{36}$ & disk (125) & 12272 & 25 & $63 \pm 2(n=4)$ & 0.02 \\
\hline Zain et al. ${ }^{36}$ & cylindrical $(125 \times 1000)$ & 404971 & 825 & $48 \pm 4(n=4)$ & not available \\
\hline Wu et al. $^{35}$ & $\operatorname{disk}(3000)$ & 7068583 & 14400 & $2 \pm 1(n=3)$ & $\sim 100$ \\
\hline Dominguez et al. ${ }^{44}$ & cylindrical $(3000 \times 4000)$ & 44767695 & 91200 & $0.34 \pm 0.001(n=10)$ & 23 \\
\hline Nieh et al. ${ }^{45}$ & disk (3000) & 7068583 & 14400 & $0.08(n=3)$ & 2 \\
\hline
\end{tabular}

${ }^{a}$ Diameter $\times$ Height. ${ }^{b}$ The surface area ratio is defined as the ratio of the electroactive surface area of other biosensors to that of the experimental biosensor.

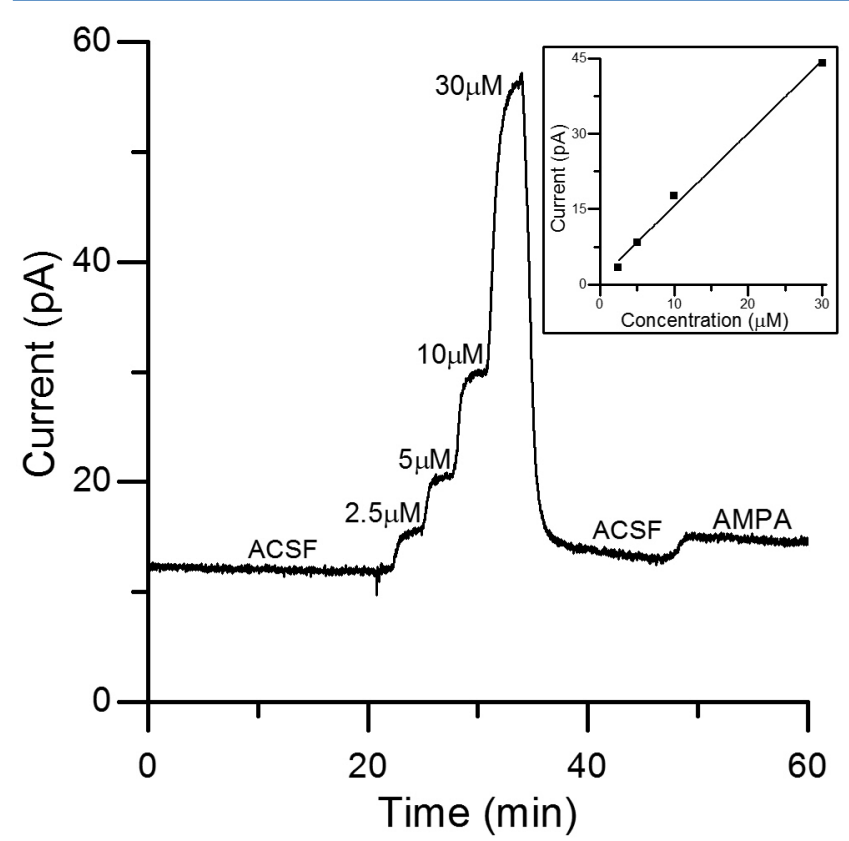

Figure 5. In vivo detection of D-serine inside the optic tectum of a Xenopus laevis tadpole. Standard solutions of D-serine were flowed into the measurement chamber to calibrate the biosensor in vivo $(t=20-$ $30 \mathrm{~min})$. Perfusion of AMPA into the chamber $(t=47 \mathrm{~min})$ stimulated release of endogenous D-serine, which was then measured locally. Inset: calibration curve obtained in vivo for $0-30 \mu \mathrm{M}$ D-serine $(n=3)$. Linear regression equation: $I(\mathrm{pA})=1.4 \times C(\mu \mathrm{M})-0.6 ; R^{2}=0.995$; $\mathrm{LOD}=0.6 \pm 0.1 \mu \mathrm{M} ; \mathrm{LOQ}=2.1 \pm 0.3 \mu \mathrm{M}$. Currents were measured at constant potential $(500 \mathrm{mV})$.

measured using an amperometric probe in the living brain. Furthermore, using the in vivo calibration completed prior to AMPA exposure, it is possible to establish that the peak level of endogenous D-serine within the CNS of tadpoles is on the order of $2.5 \pm 0.3 \mu \mathrm{M}(n=5)$. This concentration falls within the linear dynamic range of the calibration curve and is above the LOQ, making it analytically valid.

Although the presence of the PPD layer significantly reduces the contribution of interferences to the recorded current, the complex sample matrix within the optic tectum could create a false-positive response. To exclude this possibility, a null biosensor, which consisted of all the same components except for the highly specific enzyme RgDAAO that underlies D-serine sensitivity, was also tested. The in vivo procedure described above was repeated with the null biosensor (see SI; Figure S3), and no elevated current response was observed after addition of either standard D-serine solutions or AMPA. This indicates that the current response obtained using the disk-shaped experimental D-serine biosensor was in fact related to oxidation of $\mathrm{D}$ serine and not other electroactive species.

\section{CONCLUSIONS}

We have successfully designed and fabricated a fully functional disk-shaped amperometric enzymatic biosensor for detection of $\mathrm{D}$-serine, useable in both in vitro and in vivo environments. Its geometry allows for increased mechanical robustness and high spatial resolution. Furthermore, the experimental disk-shaped biosensor exhibited a higher sensitivity to D-serine than any other previously reported amperometric probe.

Although its use in vivo was demonstrated here in the optic tectum of tadpoles, this biosensor could be readily adapted to applications for the study of D-serine contributions to neuroplasticity in acute brain slices or in vivo. Its small size allows for spatially precise measurements not possible using currently available amperometric probes. For example, it could be used to measure D-serine availability and release in different layers of the cerebral cortex. Its small profile would also make it suitable for simultaneous calcium imaging to better assess the temporal relationship of glial calcium transients to gliotransmitter release and the signaling cascades contributing to Dserine release. Potential applications for dissociated cultured cells might include a systematic mapping of the subcellular locations of presumptive sites of D-serine release from astrocytes and neurons. Overall, this biosensor provides a useful tool for detection of D-serine in any experimental situation where an amperometric probe could be applied.

\section{ASSOCIATED CONTENT}

\section{Supporting Information}

Additional information as noted in the text. This material is available free of charge via the Internet at http://pubs.acs.org.

\section{AUTHOR INFORMATION}

\section{Corresponding Authors}

*E-mail: (J.M.) janine.mauzeroll@mcgill.ca. Fax: +1-514-3983797. Tel.: 514-398-3898.

*E-mail: (E.S.R.) edward.ruthazer@mcgill.ca. Fax: +1-514-3986547. Tel.: 514-398-4022.

\section{Author Contributions}

${ }^{\perp}$ D.P and A.K contributed equally to this work. The manuscript was written through contributions of all authors. All authors have given approval to the final version of the manuscript. 


\section{Funding}

\section{Notes}

The authors declare no competing financial interest.

\section{ACKNOWLEDGMENTS}

D.P., A.K., E.S.R, and J.M. acknowledge financial support from the Natural Sciences and Engineering Research Council of Canada (NSERC), CREATE: Training Program in Neuroengineering, and the Canadian Foundation for Innovation (CFI). M.R.V.H. held a NSERC Banting Postdoctoral Fellowship. E.S.R. is supported by an operating grant from the Canadian Institutes of Health Research. L.P. acknowledges support from MIUR Fondo di Ateneo per la Ricerca and from Consorzio Interuniversitario per le Biotecnologie (CIB). We thank Dr. Laura Caldinelli for her help in enzyme preparation.

\section{REFERENCES}

(1) Mothet, J. P.; Parent, A. T.; Wolosker, H.; Brady, R. O., Jr.; Linden, D. J.; Ferris, C. D.; Rogawski, M. A.; Snyder, S. H. Proc. Natl. Acad. Sci. U.S.A. 2000, 97, 4926-4931.

(2) Papouin, T.; Ladépêche, L.; Ruel, J.; Sacchi, S.; Labasque, M.; Hanini, M.; Groc, L.; Pollegioni, L.; Mothet, J.-P.; Oliet, S. H. R. Cell 2012, 150, 633-646.

(3) Nicoll, R. A.; Kauer, J. A.; Malenka, R. C. Neuron 1988, 1, 97103.

(4) Paoletti, P.; Bellone, C.; Zhou, Q. Nat. Rev. Neurosci. 2013, 14, 383-400.

(5) Moghaddam, B.; Javitt, D. Neuropsychopharmacology 2012, 37, 415.

(6) Hashimoto, K.; Fukushima, T.; Shimizu, E.; Komatsu, N.; Watanabe, H.; Shinoda, N.; Nakazato, M.; Kumakiri, C.; Okada, S.; Hasegawa, H.; Imai, K.; Iyo, M. Arch. Gen. Psychiatry 2003, 60, 572576.

(7) Milnerwood, A. J.; Raymond, L. A. Trends Neurosci. 2010, 33, 513-523.

(8) Spalloni, A.; Nutini, M.; Longone, P. Biochim. Biophys. Acta 2013, $1832,312-322$.

(9) Sasabe, J.; Chiba, T.; Yamada, M.; Okamoto, K.; Nishimoto, I.; Matsuoka, M.; Aiso, S. EMBO J. 2007, 26, 4149-4159.

(10) Curtis, D. R.; Johnston, G. A. Ergeb. Physiol., Biol. Chem. Exp. Pharmakol. 1974, 69, 97-188.

(11) Johnson, J. W.; Ascher, P. Nature 1987, 325, 529-531.

(12) Van Horn, M. R.; Sild, M.; Ruthazer, E. S. Front. Cell. Neurosci. 2013, 7, 39.

(13) Martineau, M.; Shi, T.; Puyal, J.; Knolhoff, A. M.; Dulong, J.; Gasnier, B.; Klingauf, J.; Sweedler, J. V.; Jahn, R.; Mothet, J. P. J. Neurosci. 2013, 33, 3413-3423.

(14) Volterra, A.; Meldolesi, J. Nat. Rev. Neurosci. 2005, 6, 626-640.

(15) Cornell-Bell, A. H.; Finkbeiner, S. M.; Cooper, M. S.; Smith, S. J. Science 1990, 247, 470-473.

(16) Panatier, A.; Vallee, J.; Haber, M.; Murai, K. K.; Lacaille, J. C.; Robitaille, R. Cell 2011, 146, 785-798.

(17) Di Castro, M. A.; Chuquet, J.; Liaudet, N.; Bhaukaurally, K.; Santello, M.; Bouvier, D.; Tiret, P.; Volterra, A. Nat. Neurosci. 2011, $14,1276-1284$.

(18) Hogerton, A. L.; Bowser, M. T. Anal. Chem. 2013, 85, 90709077.

(19) Rosenberg, D.; Kartvelishvily, E.; Shleper, M.; Klinker, C. M.; Bowser, M. T.; Wolosker, H. FASEB J. 2010, 24, 2951-2961.

(20) Goodnough, D. B.; Lutz, M. P.; Wood, P. L. J. Chromatogr. B: Biomed. Sci. Appl. 1995, 672, 290-294.

(21) Sethuraman, R.; Krishnamoorthy, M. G.; Lee, T. L.; Liu, E. H. C.; Chiang, S.; Nishimura, W.; Sakai, M.; Minami, T.; Tachibana, S. Clin. Chem. 2007, 53, 1489-1494.

(22) Klinker, C. C.; Bowser, M. T. Anal. Chem. 2007, 79, 87478754.
(23) Zhao, S.; Yuan, H.; Xiao, D. J. Chromatogr. B: Anal. Technol. Biomed. Life Sci. 2005, 822, 334-338.

(24) Masson, J. F.; Kranz, C.; Mizaikoff, B.; Gauda, E. B. Anal. Chem. 2008, 80, 3991-3998.

(25) Kulagina, N. V.; Shankar, L.; Michael, A. C. Anal. Chem. 1999, $71,5093-5100$.

(26) Pollegioni, L.; Langkau, B.; Tischer, W.; Ghisla, S.; Pilone, M. S. J. Biol. Chem. 1993, 268, 13850-13857.

(27) Hashimoto, A.; Nishikawa, T.; Hayashi, T.; Fujii, N.; Harada, K.; Oka, T.; Takahashi, K. FEBS Lett. 1992, 296, 33-36.

(28) Maucler, C.; Pernot, P.; Vasylieva, N.; Pollegioni, L.; Marinesco, S. ACS Chem. Neurosci. 2013, 4, 772-781.

(29) Pernot, P.; Mothet, J. P.; Schuvailo, O.; Soldatkin, A.; Pollegioni, L.; Pilone, M.; Adeline, M. T.; Cespuglio, R.; Marinesco, S. Anal. Chem. 2008, 80, 1589-1597.

(30) Fantinato, S.; Pollegioni, L.; Pilone, M. S. Enzyme Microb. Technol. 2001, 29, 407-412.

(31) Schell, M. J.; Molliver, M. E.; Snyder, S. H. Proc. Natl. Acad. Sci. U.S.A. 1995, 92, 3948-3952.

(32) Mothet, J. P.; Pollegioni, L.; Ouanounou, G.; Martineau, M.; Fossier, P.; Baux, G. Proc. Natl. Acad. Sci. U.S.A. 2005, 102, 56065611.

(33) Sullivan, S. J.; Miller, R. F. J. Neurochem. 2010, 115, 1681-1689.

(34) Van-Horn, M. R.; Sild, M.; Ruthazer, E. S. Front. Cell. Neurosci. 2013, DOI: 10.3389/fncel.2013.00039.

(35) Wu, X.; Van Wie, B. J.; Kidwell, D. A. Biosens. Bioelectron. 2004, 20, 879-886.

(36) Zain, Z. M.; O’Neill, R. D.; Lowry, J. P.; Pierce, K. W.; Tricklebank, M.; Dewa, A.; Ghani, S. A. Biosens. Bioelectron. 2010, 25, 1454-1459.

(37) Vasylieva, N.; Barnych, B.; Meiller, A.; Maucler, C.; Pollegioni, L.; Lin, J. S.; Barbier, D.; Marinesco, S. Biosens. Bioelectron. 2011, 26, 3993-4000.

(38) Lopez-Gallego, F.; Betancor, L.; Mateo, C.; Hidalgo, A.; AlonsoMorales, N.; Dellamora-Ortiz, G.; Guisan, J. M.; Fernandez-Lafuente, R. J. Biotechnol. 2005, 119, 70-75.

(39) Migneault, I.; Dartiguenave, C.; Bertrand, M. J.; Waldron, K. C. BioTechniques 2004, 37, 790-802.

(40) Yuqing, M.; Jianrong, C.; Xiaohua, W. Trends Biotechnol. 2004, 22, 227-231.

(41) Dai, Y.-Q.; Zhou, D.-M.; Shiu, K.-K. Electrochim. Acta 2006, 52, 297-303.

(42) Killoran, S. J.; O’Neill, R. D. Electrochim. Acta 2008, 53, 73037312 .

(43) Michael, A. C.; Borland, L. Electrochemical Methods for Neuroscience; Taylor \& Francis: Boca Raton, FL, 2010.

(44) Domínguez, R.; Serra, B.; Reviejo, A. J.; Pingarrón, J. M. Anal. Biochem. 2001, 298, 275-282.

(45) Nieh, C. H.; Kitazumi, Y.; Shirai, O.; Kano, K. Biosens. Bioelectron. 2013, 47, 350-355. 